\title{
O uso de psicofármacos em jovens universitários
}

\author{
Psychotropic drugs use in young university students \\ El uso de droga psicotrópica en jóvenes universitarios
}

\section{Resumo}

O adoecimento psíquico em jovens universitários e o suo de psicofármacos nesta população tem ganhado destaque em estudos atuais. Os diagnósticos de transtornos mentais comuns, como ansiedade e a depressão são encontrados na maioria dos estudantes, e quando não tratados esses sintomas podem desencadear prejuízos maiores. Portanto, este estudo objetivou realizar um levantamento bibliográfico dos últimos cinco anos de estudos relacionados ao uso de psicofármacos no ensino superior. A busca foi realizada nas bases da CAPES, Scielo e Google Acadêmico, utilizando as palavras-chaves: psicofármacos; acadêmicos; farmácia; saúde do estudante e prevalência. Foram selecionados dez estudos entre artigos, teses e dissertações. Observou-se que a prevalência de adoecimento mental está no sexo feminino. Grande parte deste público faz uso de medicamentos antidepressivos, calmantes e estimulantes para tratar sintomas depressivos, ansiedade, dificuldade para dormir e para aumento do rendimento acadêmico. Muitos estudantes fazem o uso por indicação de amigos ou terceiros sem prescrição médica. O estresse cotidiano, as exigências acadêmicas, conciliar trabalho e estudo e condições socioeconômica desfavoráveis contribuem para o adoecimento psíquico.

Palavras-chave: Psicofármacos; Universitários; Medicamento; Adoecimento mental.

\begin{abstract}
Psychic illness in university students and psychotropic drugs use in this population has gained prominence in current studies. Common mental disorders diagnoses such as anxiety and depression are found in most students, and when untreated, these symptoms can lead to greater harm. Therefore, this study aimed to carry out the last five years bibliographic studies related to the use of psychotropic drugs in higher education. The search was carried out in CAPES, Scielo and Google Academic databases, using the keywords: psychopharmaceuticals; academics; drugstore; student health and prevalence. Ten studies were selected among articles, theses and dissertations. It was observed that the prevalence of mental illness is in females. A large part of this public uses antidepressant, calming and stimulant medications to treat depressive symptoms, anxiety, difficulty in sleeping and to increase academic performance. Many students use it on the recommendation of friends or third parties without a prescription. Daily stress, academic demands, reconciling work, study and unfavorable socioeconomic conditions contribute to mental illness.
\end{abstract}

Keywords: Psychopharmaceuticals; College students; Medicine; Mental illness.

\section{Resumen}

La enfermedad psíquica en estudiantes universitarios y el uso de psicofármacos en esta población ha ganado protagonismo en los estudios actuales. En la mayoría de los estudiantes se encuentran diagnósticos de trastornos mentales comunes, como ansiedad y depresión, y si no se tratan, estos síntomas pueden provocar un daño mayor. Por tanto, este estudio tuvo como objetivo realizar un relevamiento bibliográfico de los últimos cinco años de estudios relacionados con el uso de psicofármacos en la educación superior. La búsqueda se realizó en las bases de datos CAPES, Scielo y Google Academic, utilizando las palabras clave: psicofármacos; académica; farmacia; salud y prevalencia de los estudiantes. Se seleccionaron diez estudios entre artículos, tesis y disertaciones. Se observó que la prevalencia de enfermedades mentales es en mujeres. Una gran parte de este público utiliza medicamentos antidepresivos, calmantes y estimulantes para tratar síntomas depresivos, ansiedad, dificultad para dormir y aumentar el rendimiento académico. Muchos estudiantes lo usan por recomendación de amigos o terceros sin receta. El estrés 
diario, las exigencias académicas, la conciliación del trabajo y el estudio y las condiciones socioeconómicas desfavorables contribuyen a la enfermedad mental.

Palabras clave: Psicofármacos; Estudiantes universitarios; Medicamento; Enfermedad mental.

\section{Introdução}

Diversos estudos apresentam dados referente ao adoecimento psíquico de jovens que estão cursando o ensino superior (Pedro, 2017, Funai, 2019, Giajacomo, 2020). Grande parte desses estudantes tem apresentado sintomas como humor deprimido, ingestão e abuso de álcool, e aumento significativo no uso de medicamentos psiquiátricos.

Para Giajacomo (2020) os psicofármacos voltados ao tratamento de depressão e ansiedade são os mais consumidos pela população jovem entre 18 à 24 anos que cursam o ensino superior em uma universidade pública do estado do Paraná. Para a autora, sujeitos que possuem planos de saúde e terem feito uso de drogas ilícitas no último mês são as características mais preponderantes para o uso de psicofármacos. Também considera como fatores positivos ao uso de medicação, jovens que estão insatisfeitos com o meio acadêmico, serem dependentes de mídias sociais e terem diagnóstico de depressão.

Segundo Pedro (2017) em uma amostra com 792 estudantes de cursos da área de saúde, a maior incidência ao uso de medicamentos psicotrópicos está no sexo feminino. Como também Giajacomo (2020) ressalta que uma das possibilidades de os homens possuírem menores índices de adoecimento psíquico é que eles possuem melhor percepção de sua saúde física e mental e menor preocupação com a imagem corporal.

Silva (2021) observou que os estudantes de graduação apresentam grau moderado de adaptação acadêmica e que pode estar relacionado com os sintomas de ansiedade. Para a autora, quanto maior o nível de ansiedade menor é o grau de adaptação acadêmica nas dimensões da carreira pessoal e interpessoal.

O sofrimento psicológico dos acadêmicos, de acordo com Santos (2020) está ligado ao desamparo institucional das necessidades psicossociais, pois os transtornos mentais comuns, como a ansiedade e a depressão são encontrados na maioria dos estudantes e a negligência a esses sintomas podem desencadear prejuízos maiores. Por exemplo, as rotinas de excesso de atividades, questões financeiras, sujeitos que conciliam trabalho e estudo, cobranças dos professores, dos familiares e de si próprio, alta carga horária de aulas, dificuldades de adaptação e o manejo do tempo para estudo e lazer, são fontes desse malestar relacionados às vicissitudes da vida universitária.

Outro fator importante é que muitos desses jovens fazem uso de psicotrópicos sem prescrição médica. Conseguem receitas com colegas ou familiares, e fazem o uso por influências de terceiros, para aumentar rendimento escolar, inibir apetite ou para tratar sintomas de ansiedade e depressão (Araujo, 2019).

Para Nogueira e Siqueira (2017), o nível de saúde mental é mais baixo entre mulheres, principalmente as que praticam pouca atividade física ou que possuem dificuldades socioeconômicas. Fesh et al (2020) buscaram avaliar a prevalência e fatores associados ao episódio depressivo maior em universitários, com ênfase na influência do meio acadêmico, área de estudo escolhida e ambiente onde o universitário está inserido. Identificaram que a maior frequência de adoecimento está no sexo feminino, de idade entre 21 e 23 anos, com histórico familiar de depressão, orientação sexual homoafetiva ou bissexual, que moravam com amigos e colegas, e cursam graduação na área de humanas. O abuso de álcool e uso de drogas ilícitas também estão associados positivamente aos sintomas de depressão maior.

Leão et al. (2018) estimaram a prevalência e os fatores associados à depressão e ansiedade em estudantes universitários da área de saúde. Constataram que na amostra de 476 participantes, 28,6\% apresentaram sintomas de depressão e 36,1\% de ansiedade. Em relação a vida acadêmica, os estudantes menos satisfeitos com o curso apresentaram quase quatro vezes maior incidência de terem depressão. Também identificaram que o relacionamento familiar e de amizades como insatisfatórios, poucas horas de sono, não realizar atividades físicas e preocupações exageradas com o futuro estão atreladas diretamente ao adoecimento psíquico. 
No estudo de Veloso et al. (2019), objetivaram identificar o nível de ideação suicida em universitários da área de saúde de uma instituição pública do estado de Piaú. Com amostra de 142 acadêmicos de medicina, 22\% apresentaram prevalência de ideação suicida, sobretudo, entre homens, solteiros e com vínculo empregatício. Os autores também associaram o uso de bebida alcoólica, tabaco, outras drogas, ter histórico de bullying e não fazer o curso desejado são fatores que aumentam as chances de ter ideação suicida.

Contudo, Souza et al. (2016), identificou em sua amostra que universitários reconhecem que a doença psíquica está atrelada a não fatores só biológicos, mas psicológicos e sociais. No entanto, quando questionados sobre a doença mental se mantem sob o discurso biomédico, ou seja, de que as enfermidades mentais devem ser encaradas como uma condição biológica, como por exemplo, os tratamentos se baseiam, principalmente no uso de medicamentos psicotrópicos, não havendo uma compreensão total e complexa dos fatores responsáveis pelo adoecimento.

A partir da complexidade do tema abordado, este estudo tem como objetivo levantar dados dos últimos anos, referente ao uso de psicofármacos em jovens universitários, afim de discutir melhor o assunto e trazer resultados atualizados sobre o uso de medicamentos e o adoecimento mental de jovens.

\section{Metodologia}

Com o intuito de buscar referências sobre a prevalência do uso de psicofármacos em jovens acadêmicos utilizou-se como metodologia a revisão bibliográfica integrativa. Para Marconi e Lakatos (2003), este tipo de pesquisa busca as publicações existentes de uma determinada área, acerca de um tema especifico em um determinado período de tempo para discorrer o que as obras discutem sobre o tema.

Todavia, a busca dos artigos ocorreu em outubro de 2021 pelas bases de dados Scielo, Periódicos Capes e Google Acadêmico. Na plataforma do Scielo foram utilizadas as palavras-chave: prevalência; psicofármacos; acadêmicos; saúde do estudante e farmácia, porém não foram encontrados nenhum estudo envolvendo essas palavras. Assim, realizou-se uma nova busca utilizando apenas as palavras 'prevalência' e 'psicofármacos' e foram encontrados 20 estudos, no qual três eram repetidos e os demais realizavam levantamento sobre a prevalência e uso de psicotrópicos, mas em outras amostras como idosos e crianças, desviando do objetivo deste estudo.

A busca realizada na plataforma de Periódicos Capes foi realizada utilizando as palavras "psicofármacos e 'acadêmicos' limitadas de 2017 até 2021. Foram encontrados 178 resultados, destes, 10 artigos eram repetidos e os demais abrangiam assuntos como psicofármacos na atenção primaria a saúde, prescrição de medicamentos na infância, uso de psicotrópicos em relação a psicoterapia, entre outros. Apenas quatro estudos foram selecionados. No entanto, uma nova busca foi realizada pelo Google Acadêmico, onde também utilizou-se as palavras-chaves: prevalência; psicofármacos; acadêmicos; saúde do estudante; farmácia para estudos dos últimos cinco anos. Foram encontrados 298 estudos, entre artigos, teses e dissertações. Dez artigos eram duplicados e sete foram selecionados para este estudo. Ao total, entre os dois periódicos, 11 trabalhos compuseram a amostra deste estudo. Os resultados desse procedimento estão descritos no Fluxograma abaixo: 
Figura 1. Fluxograma da revisão.

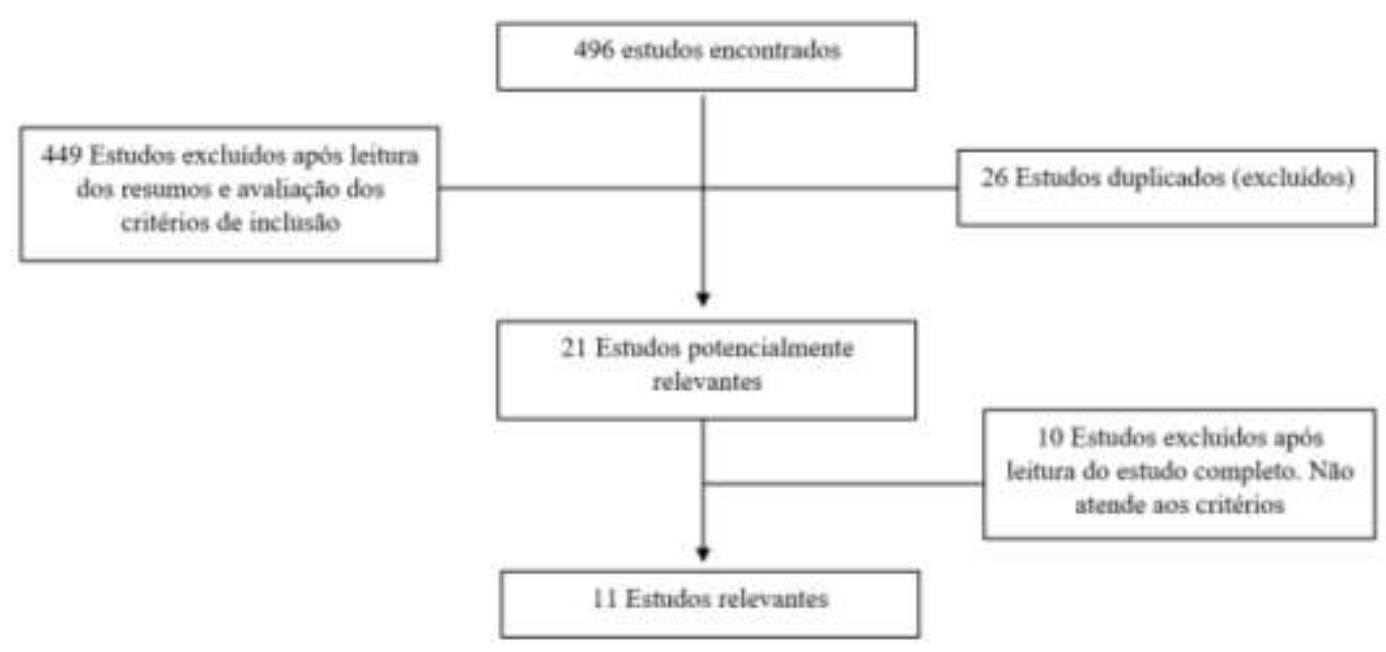

Fonte: Autores.

No Quadro abaixo segue os estudos selecionados com sua respectiva autoria, tipo de estudo, ano e as palavras-chave utilizadas.

Quadro 1. Estudos selecionados.

\begin{tabular}{|c|c|c|c|}
\hline Autor/Título/Ano & Palavras-chaves & Tipo & Ano \\
\hline $\begin{array}{c}\text { Bauchrowitz, C et al. Prevalência do uso de psicofármacos } \\
\text { por acadêmicos: Efeitos do processo de graduação. }\end{array}$ & tal; ansiedade; depressão & Artigo & 2019 \\
\hline
\end{tabular}

Silvano, L. V. P; Tomasi, C. D. Prevalência e fatores associados à utilização de psicofármacos entre acadêmicos da área da saúde.

saúde mental; psicofármacos; utilização Artigo

Araujo, F, L, L, A. Investigação do uso de psicofármacos entre estudantes.

Psicotrópicos; Estudantes de medicina;

Estudantes de odontologia; Saúde Dissertação menta

Souza, R. C. et al. O uso de antidepressivos em estudantes da área da saúde.

Filho, M. L. V.; Sperandio, G.; Ferreira, E. D. F.; Análise da prevalência de uso de antidepressivos e psicoestimulantes e seus efeitos sobre acadêmicos de medicina de uma universidade da região noroeste do Paraná.

Luna, I. S., Grigoli Dominato, A. A., Ferrari, F., da Costa, A. L., Pires, A. C., \& Ximendes, G. da S. (2018). Consumo de psicofármacos entre alunos de medicina do primeiro e sexto ano de uma universidade do estado de São Paulo.

Coimbra, Marla Brenda Pires; Araújo, Renata Aparecida Faria de. Avaliação do uso de antidepressivos e ansiolíticos por acadêmicos do curso de enfermagem.

Brito, J. R.; Silva, P. R. Consumo de ansiolíticos e antidepressivos: Uma análise sobre o uso entre estudantes de Medicina.
Estresse; Antidepressivos;

Psicoestimulantes; Acadêmicos de Artigo 2019 medicina; Prevalência

Psicotrópicos, Estudantes de Medicina, Educação de Graduação em Medicina, Medicalização, Educação Superior.

Artigo 2018

Ansiolíticos, antidepressivos, saúde mental, curso de enfermagem

Artigo

Psicotrópicos, ansiolíticos, antidepressivos, universitários, saúde.
Artigo 
Terra Junior, A. T. Perfil dos universitários da área da saúde quanto ao uso de substâncias psicoativas na cidade de Ariquemes.

\section{Aprimoramento cognitivo, farmacológico, consumo de álcool na faculdade, medicalização,} metilfenidato, psicofármacos
Tese

2018

Estudantes, psicotrópicos, uso indiscriminado

Artigo associados ao consumo de benzodiazepínicos por estudantes de enfermagem e farmácia de uma Faculdade Particular do Sul da Bahia.

Oliveira, K. A. de. Et al.Prevalência do uso de drogas antidepressivas por estudantes da área da saúde no Brasil.
Artigo
Estudantes de Ciências da Saúde; Antidepressivos; Ansiolíticos; Depressão.

Fonte: Autores.

\section{Discussão}

Com o objetivo de demonstrar o perfil sociodemográfico e a prevalência do uso dos psicofármacos entre acadêmicos de uma universidade pública localizada no estado do Paraná, Bauchrowitz et. al (2019) realizaram um estudo de cunho transversal por meio da aplicação de um questionário eletrônico, cujo a amostra foram 431 estudantes do primeiro e último ano de graduação, sendo analisados dois setores de Ciências Biológicas e da Saúde (setor A) e Ciências Sociais Aplicadas (setor B). a maioria era do sexo feminino correspondendo $65,6 \%$ com média de idade de 21,5 anos. A prevalência do uso de medicamentos psicotrópicos foi de 22,3\%, sendo o Escitalopram (15\%), Sertralina (14\%) e Fluoxetina (13\%) os mais usados. A porção dos acadêmicos que já haviam feito tratamento para ansiedade e depressão e que ainda estavam em tratamento é de $59,4 \%$, desses $31,3 \%$ encerraram o tratamento por conta própria; $16,7 \%$ encerraram com acompanhamento médico; $21,9 \%$ continuam o tratamento e 30,1\% não informaram. A classe dos benzodiazepínicos também foi destaque na pesquisa, o clonazepam e alprazolam juntos somam o total de $15 \%$ dos medicamentos utilizados. Os fitoterápicos foram mencionados devido ao fácil acesso e o conhecimento dos estudantes, o Passiflora incarnata e/ou Valeriana officinalis foram os principais em utilização $(12,3 \%)$.

Segundo os autores, a respeito de informações sobre os psicofármacos que são capazes de avaliar a adesão ao tratamento, por exemplo, nome do medicamento, posologia e concentração, 52,4\% dos acadêmicos souberam informar todos os aspectos em relação ao seu tratamento, $37,1 \%$ conseguiram responder pelo menos um dos itens questionados e $10,5 \%$ não responderam nenhum. A não-adesão não intencional também foi observada tendo 44,8\% de frequência, desses $64,6 \%$ relataram esquecimento na tomada dos medicamentos, $26 \%$ não informaram e 9,4\% não apresentaram dificuldades (Bauchrowitz, et. al, 2019).

Concomitante, Silvano et. al (2019), objetivaram identificar a prevalência e fatores associados a utilização dos psicofármacos entre acadêmicos da área da saúde. Fizeram parte de sua amostra 541 acadêmicos com média de 22 anos. A porcentagem de alunos que passaram a utilizar os medicamentos após o ingresso no ensino superior foi de $63,9 \%$, em contrapartida os outros 36,1\% relataram iniciar antes mesmo de seu ingresso. O sexo feminino apresenta prevalência de 23,3\% em relação ao uso de psicofármacos contra $15,5 \%$ do sexo masculino. Observaram também que ao final dos cursos da área da saúde a recorrência pelos medicamentos psicotrópicos se acentua com 23,2\%, considerando que a 22,3\% é a média global.

A autora Coimbra (2020) também traz dados que reforçam o fato de que a maior porção dos acadêmicos passam a fazer uso de psicotrópicos após o ingresso no ensino superior, sua pesquisa com 79 acadêmicos elucidou que esse evento ocorre com 61,54\% dos alunos que fazem o uso dos psicofármacos; $16 \%$ do total de graduandos usam 
ansiolíticos/antidepressivos. Os dados sociodemográficos levantados apontam que 93,67\% são do feminino e 6,33\% do masculino; 36,71\% dividem a atenção entre os estudos e o trabalho em uma jornada dupla; os que dizem ser casados são 18,99\% e 11,39\% que possuem filhos. Outros fatores como ter plano de saúde e renda familiar, 50,63\% não possuem plano de saúde e apenas 12,66\% relatam ter renda média acima de cinco salários mínimos.

Araujo (2019), em uma amostra censitária de 1.111 estudantes dos cursos de odontologia e medicina de duas universidades, uma pública e outra privada, na cidade de Maceió-AL identificou que 14,7\% relataram terem feito uso de psicofármacos no último mês e quando questionados ao uso durante a vida, o percentual sobe para 36,7\%. Este estudo não identificou uma diferença estatística relevante no que tange o uso dos psicofármacos entre os sexos. Outro dado importante, é que $21,5 \%$ adquiriram os medicamentos com familiares e, 12,9\% sem receituário médico, caracterizando alta prevalência da automedicação. Destes, 90,8\% afirmaram ter consciência do risco que podem causar o uso desses medicamentos.

A pesquisa de Filho, Sperandio e Ferreira (2019) verificou a prevalência, causas e consequências do uso de ansiolíticos e psicoestimulantes. Utilizaram de um um questionário online com 96 estudantes universitários. Destes, 46\% revelaram já terem utilizado medicamentos antidepressivos. Quanto a qualidade de sono dos acadêmicos $63 \%$ apresentam sono irregular e 17,7\% utilizam medicação para dormir. O maior motivador do consumo de psicoestimulantes é a redução do sono com 78,7\%, o que acarreta na piora da qualidade do repouso e sono, e o segundo motivo mais relevante é a melhoria na concentração com $65,6 \%$. Dentro os usuários de psicoativos desta pesquisa $85,2 \%$ não faz seu uso por indicação médica, além de que dos 61 estudantes, 45 deles dizem sentir efeitos adversos dos medicamentos, como agitação e insônia.

Em relação ao uso de antidepressivos e psicoestimulantes por jovens acadêmicos, Souza et. al (2021), identificou em 200 alunos dos cursos de Enfermagem, Farmácia, Nutrição e Psicologia que 46,8\% fazem uso desses medicamentos. A maior prevalência está em jovens de 18 à 24 anos. A Fluoxetina é o fármaco mais usado, seguido do Citalopram e Sertralina. Da amostra, 77,4\% revelam não terem recebido orientação do Farmacêutico quanto a administração dos psicofármacos.

Luna et. al (2017), realizou uma pesquisa comparando a utilização do uso de psicofármacos com dois grupos de estudantes de medicina, cada grupo com 100 participantes, sendo um grupo com acadêmicos do primeiro ano e o outro com estudantes do sexto ano. Observaram que o uso desses medicamentos é de $23 \%$ no primeiro ano e $50 \%$ no sexto. De acordo com o sexo, a amostra do primeiro ano prevaleceu o uso em $70 \%$ das mulheres, enquanto no último ano foi de $62 \%$ dos homens. Os autores concluem que a busca por psicofármacos é significativamente maior nos acadêmicos que estão no fim do curso de graduação.

Brito e Silva (2021), fizeram um levantamento em torno do uso de ansiolíticos e antidepressivos por acadêmicos de Medicina, foram enviados 870 formulários e após os critérios de inclusão, que eram serem estudantes de medicina e serem maiores de idade, 99,4\% da amostra foi considerada. A Faixa etária maior é dos 22 aos 25 anos (44\%) e predomina-se o sexo feminino. Dos alunos que utilizam os psicofármacos, $29,4 \%$ fazem o uso atualmente e 17,1\% já recorreram ao uso, mas não o fazem mais e $36 \%$ relataram nunca terem feito nenhum tratamento relacionado aos psicotrópicos. O tempo de uso dos medicamentos por mais de um ano representa 47,87\% dos estudantes, de seis meses a um ano 10,6\%, dois a seis meses $9.8 \%$ e $3,6 \%$ menos de um mês. A associação dos medicamentos foi averiguada pelos pesquisadores, 58,3\% estão em monoterapia, ou seja, uso de apenas um medicamento e $41,7 \%$ em terapia combinada.

Um dos objetivos de seu estudo de tese Junior (2018), buscou avaliar o consumo de medicamentos não prescritos, 518 alunos de diversas áreas fizeram parte de sua amostra, a maior parte do sexo feminino $68,5 \%$ e $70,7 \%$ tem entre 18 e 23 anos. $\mathrm{O}$ autor aferiu os fatores que influenciam os acadêmicos a utilizarem o metilfenidato, utilizado principalmente por prescrição médica para o tratamento do TDAH. De acordo com a pesquisa os estudantes apresentam mais chances de consumir o metilfenidato quando "curiosidade" 11 vezes mais; “enfrentar situações desagradáveis" 5,7 vezes mais; "pressão em torno de mim" apresenta 35 vezes mais chances; e "influência de amigos" menos de 1 vez mais chances. Quanto ao uso de 
antidepressivos apresentam mais chances de consumi-los quando "curiosidade" 2,3 vezes; "pressão em torno de mim" 3 vezes.

Ribeiro, Rodrigues e Duarte (2017) fizeram um levantamento acerca do uso de benzodiazepínicos por acadêmicos dos cursos de farmácia e enfermagem. A amostra foi composta por 135 alunos de enfermagem e 249 alunos de farmácia, ambos os cursos o sexo feminino foi a maioria com $66,7 \%$ e 51,1\% respectivamente. Dos graduandos de enfermagem $11,1 \%$ já fizeram o uso da classe dos benzodiazepínicos, o medicamento mais frequente é o Diazepam com 40\%, os principais motivadores de seu uso são ansiedade $(26,7 \%)$ e falta de sono (13,3), o Clonazepam e Lorazepam também aparecem com 33,3\% e 6,7\% de frequência cada. Os que cursavam farmácia apresentem uma prevalência de 18,1\% de uso dos benzodiazepínicos, o clonazepam é o mais utilizado com $40 \%$, a falta de sono $(24,4)$, ansiedade $(20 \%)$, preocupação $(4,4 \%)$ e a dor $(2,2 \%)$ são os principais motivos do uso e logo atrás o Diazepam (24,4\%) e Alprazolam (4,4\%) também foram mencionados.

Por fim, em uma pesquisa de revisão de literatura realizada por Oliveira et. al (2021) objetivou-se verificar a prevalência do uso de drogas antidepressivas por estudantes da área da saúde. Pôde-se constatar que o alto nível de estresse e o surgimento de sintomas depressivos está diretamente atrelado as cargas horárias exaustivas dos cursos de graduação, às fragilidades familiares e suas redes de apoio social. Esses dados colaboram para a necessidade de minimizar o sofrimento psíquico dos estudantes por meio de estratégias de prevenção em saúde mental por parte das instituições.

\section{Considerações Finais}

De acordo com o levantamento realizado pode-se observar que um número significativamente alto de jovens universitários tem buscado o uso de psicofármacos para o tratamento de sintomas psiquiátricos. Os medicamentos mais usados são antidepressivos, calmantes, hipnóticos e estimulantes. O uso está atrelado a sintomas de depressão, ansiedade, dificuldade para dormir e para aumentar rendimento acadêmico. O maior índice de uso desses medicamentos é no sexo feminino. Situações como estresse cotidiano, cobranças acadêmicas, conciliar os estudos e o trabalho e condições socioeconômicas favorecem para o adoecimento mental e o aumento a pela busca dos fármacos como alternativa de tratamento.

Contudo, observamos a necessidade de novos estudos sobre o adoecimento mental em jovens universitários, bem como pesquisas que abordem maneiras de lidar com o adoecimento psíquico dentro da universidade oferecendo assistência profissional adequada, evitando assim automedicação e todas as problemáticas envolvidas nessa prática.

\section{Referências}

Araujo, A. Felisbela, L. L. (2019). Investigação sobre o uso de psicofármacos entre estudantes universitários. 2019. 85 f. Dissertação (Mestrado) - Curso de Pesquisa em Saúde, Centro Universitário Cesmac, Maceió, AL. https://ri.cesmac.edu.br/handle/tede/805.

Bauchrowitz, C. et. al. (2019). Prevalência do uso de psicofármacos por acadêmicos: Efeitos do processo de graduação. Brazilian Journa of Development, 5(11). https://www.brazilianjournals.com/index.php/BRJD/article/view/4609.

Brito, J. R., \& Silva, P. R. (2021). Consumo de ansiolíticos e antidepressivos: Uma análise sobre o uso entre estudantes de Medicina. Trabalho de Conclusão de Curco - TCC, curso de Ciências Biológicas, Pontifícia Universidade Católica de Goiás. https://repositorio.pucgoias.edu.br/jspui/handle/123456789/2092.

Coimbra, M. B. P., \& Araújo, R. A. F. (2020). Avaliação do uso de antidepressivos e ansiolíticos por acadêmicos do curso de enfermagem. 35 f. Trabalho de Conclusão de Curso (Graduação em Enfermagem) - Universidade Federal de Mato Grosso, Instituto de Ciências Exatas e Naturais, Rondonópolis. https://bdm.ufmt.br/handle/1/1762.

Filho, M. L. V., \& Sperandio, G.; Ferreira, E. D. F. (2019). Análise da prevalência de uso de antidepressivos e psicoestimulantes e seus efeitos sobre acadêmicos de medicina de uma universidade da região noroeste do Paraná. XI EPCC - Encontro Internacional de Produção Científica. http://rdu.unicesumar.edu.br/handle/123456789/3392.

Flesch, B. D.; Houvèssou, G. M.; Munhoz, T. N.; Fassa, A. G. (2020). Episódio depressivo maior entre universitários do sul do Brasil. Revista de Saúde Pública, 54(11). doi: https://doi.org/10.11606/s1518-8787.2020054001540

Funai. A. (2019). Comportamentos de saúde, sofrimento mental e padrão de consumo de álcool entre estudantes universitários. Tese (Doutorado em Ciências) - Escola de Enfermagem de Ribeirão Preto, Universidade de São Paulo, Ribeirão Preto. https://rd.uffs.edu.br/handle/prefix/3352. 
Research, Society and Development, v. 10, n. 17, e79101724472, 2021

Gianjacomo, Telma Regina Fares. Caracterização do consumo de medicamentos psicofármacos por estudantes de uma universidade pública. (2020). Dissertação Mestrado em Ciências Farmacêuticas - Universidade Estadual de Londrina. http://www.bibliotecadigital.uel.br/document/?code=vtls000232096.

Leão, A. M. et al. (2017). Prevalência e Fatores Associados à Depressão e Ansiedade entre Estudantes Universitários da Área da Saúde de um Grande Centro Urbano do Nordeste do Brasil. Revista Brasileira de Educação Médica [online], 42(4), 2018. doi: https://doi.org/10.1590/1981-52712015v42n4RB20180092.

Luna, I. S.; et. al. (2018). Consumo de psicofármacos entre alunos de medicina do primeiro e sexto ano de uma universidade do estado de São Paulo. Colloquium Vitae, 10(1). https://revistas.unoeste.br/index.php/cv/article/view/2167.

Nogueira, M. J.; Barros, L.; Sequeira, C. (2017). A Saúde Mental em Estudantes do Ensino Superior: Relação com o género, nível socioeconómico e os comportamentos de saúde. Revista Portuguesa de Enfermagem de Saúde Mental, Porto. doi: https://doi.org/10.19131/rpesm.0167.

Oliveira, K. A., et al. (2021). Prevalence of antidepressant drug use by health students in Brazil. Research, Society and Development, 10(11). https://rsdjournal.org/index.php/rsd/article/view/19641.

Pedro, C. M. P. (2017). Distúrbios psíquicos menores em estudantes universitários da área da saúde. Dissertação de Mestrado Ciências da Saúde Programa de Pós-graduação em Enfermagem - Universidade Federal de Santa Maria. https://repositorio.ufsm.br/handle/1/20457.

Ribeiro, B. S., Rodrigues, R. L. A., \& Duarte, S. F. P. (2017). Prevalência e Fatores Associados com o Consumo de Benzodiazepínicos por Acadêmicos de Enfermagem e Farmácia de uma Faculdade Particular do Sudoeste da Bahia. Revista de Psicologia, 11(38), p. 166-176. https://idonline.emnuvens.com.br/id/article/view/887.

Silva, A. C. S. et al. (2021). Relação entre vivência acadêmica e ansiedade em estudantes universitários. Revista Contextos Clínicos, 14(2). http://revistas.unisinos.br/index.php/contextosclinicos/article/view/23260.

Silvano, L. V. P. \& Tomasi, C. D. (2019). Prevalência e fatores associados à utilização de psicofármacos entre acadêmicos da área da saúde. Trabalho de Conclusão Curso de Enfermagem, da Universidade do Extremo Sul Catarinense, UNESC. http://repositorio.unesc.net/handle/1/8069.

Souza, D. C. (2017). Condições emocionais de estudantes universitários: estresse, depressão, ansiedade, solidão e suporte social. Dissertação (Mestrado em Psicologia) - Programa de Pós-Graduação em Psicologia, Universidade Federal do Triângulo Mineiro, Uberaba.

Souza, R. C. et. al. (2021). O uso de antidepressivos em estudantes da área da saúde. Brazilian Journa of Development, 7(4). https://www.brazilianjournals.com/index.php/BRJD/article/view/28588.

Terra Junior, André Tomaz. (2019). Perfil dos universitários da área da saúde quanto ao uso de substâncias psicoativas na cidade de Ariquemes. Tese (Doutorado em Clínica Cirúrgica) - Faculdade de Medicina de Ribeirão Preto, Universidade de São Paulo, Ribeirão Preto https://teses.usp.br/teses/disponiveis/17/17137/tde-20032019-161250/pt-br.php.

Veloso, L. U. P., et al. (2019). Ideação suicida em universitários da área da saúde: prevalência e fatores associados. Revista Gaúcha de Enfermagem [online], 40. doi: https://doi.org/10.1590/1983-1447.2019.20180144 